\title{
An Experience of Multiple Hematomas in a Coronavirus Disease-19 Patient Administered with ART-I23 and Heparin
}

\author{
Hideto Shiraki ${ }^{1}$ \\ Koji Morishita' \\ Mitsuhiro Kishino ${ }^{2}$ \\ Keita Nakatsutsumi ${ }^{1}$ \\ Koichiro Kimura ${ }^{2}$ \\ Tsuyoshi Shirai $^{3}$ \\ Masahiro Ishizuka (D) ${ }^{3}$ \\ Yasunari Miyazaki ${ }^{3}$ \\ Junichi Aiboshi' \\ Yasuhiro Otomo' \\ 'Department of Acute Critical Care and \\ Disaster Medicine, Tokyo Medical and \\ Dental University Hospital of Medicine, \\ Tokyo, Japan; ${ }^{2}$ Department of Diagnostic \\ Radiology, Tokyo Medical and Dental \\ University Hospital of Medicine, Tokyo, \\ Japan; ${ }^{3}$ Department of Respiratory \\ Medicine, Tokyo Medical and Dental \\ University Hospital of Medicine, Tokyo, \\ Japan
}

Background: Anticoagulant therapy for patients with severe coronavirus disease (COVID19) pneumonia is considered to improve the hypercoagulable and inflammatory state. However, bleeding complications should also be considered.

Case Presentation: A 77-year-old man with a history of falls was diagnosed with COVID19. Owing to his severe condition, he was intubated and transferred to our hospital for intensive care. Favipiravir, tocilizumab, unfractionated heparin, and ART-123 were administered to treat COVID-19 and manage the antithrombotic prophylaxis for paroxysmal atrial fibrillation (Af). On the 6th day after admission, a hematoma was noted on the left chest wall. Computed tomography (CT) revealed multiple hematomas, including hematomas on his chest wall and obturatorius internus muscle. Emergency angiography transcatheter embolization (TAE) was performed. The patient was transferred to another hospital 23 days after TAE, without complications.

Conclusion: Our findings show that anticoagulation therapy and a history of falls induced multiple hematomas in a COVID-19 patient and that the condition was managed with TAE. When anticoagulants are considered in the management of Af and COVID-19 associated coagulopathy, it is necessary to closely monitor potential bleeding complications.

Keywords: anticoagulation, complication, COVID-19, hematoma, TAE

\section{Introduction}

The coronavirus disease (COVID-19) outbreak is an ongoing pandemic. Currently, more than 12 million confirmed cases of infection have been reported. ${ }^{1}$ COVID-19 is known to induce a hypercoagulable state. As such, anticoagulant therapy has been recommended for selected patients diagnosed with COVID-19. ${ }^{2}$ We encountered a case of anticoagulation therapy that induced multiple hematomas, including a massive chest wall and obturatorius internus muscle hematoma, in an elderly patient with COVID-19. This was an indication for emergency transcatheter embolization (TAE) in the branches of the axillary arteries. This case report describes our experience with the use of emergency TAE for anticoagulation therapy-induced multiple hematomas in a COVID-19 patient.

Department of Acute Critical Care and Disaster Medicine, Tokyo Medical and Dental University Hospital of Medicine, I-5-45 Yushima, Bunkyo-ku, Tokyo, II38510 , Japan

Tel +8|-3-38|3-6| II

Fax +8I-3-5803-0I37

Email morishita.accm@tmd.ac.jp

\section{Case Presentation}

A 77-year-old man was presented to the hospital after sustaining a fall due to fever and weakness. On admission, a routine reverse transcription polymerase chain 
reaction (RT-PCR) test was performed. A diagnosis of COVID-19 caused by severe acute respiratory syndrome coronavirus 2 infection was confirmed. Chest CT showed typical signs of pneumonia, including bilateral groundglass opacities. The patient reported worsening of his respiratory symptoms during the preceding few days. Owing to his severe condition, he was intubated and transferred to our hospital's intensive care unit (ICU) on day 5 of hospitalization. On arrival at our hospital, his vital signs were as follows: blood pressure, 124/73 $\mathrm{mmHg}$; heart rate, 63 beats/min with sinus rhythm; $\mathrm{SpO}_{2}$ $90 \%\left(\mathrm{FiO}_{2}, 50 \%\right)$; and body temperature, $38.0{ }^{\circ} \mathrm{C}$; laboratory tests showed white blood cell (WBC) count, $6900 / \mu \mathrm{L}$; hemoglobin $(\mathrm{Hb}), 11.3 \mathrm{~g} / \mathrm{dL}$; platelet, $197 \times 103 / \mu \mathrm{L}$; activated partial thromboplastin time (aPTT), 29 sec; prothrombin time (PT), 1.09; D-dimer, $1.93 \mu \mathrm{g} / \mathrm{mL}$; and CRP, $12.74 \mathrm{mg} / \mathrm{dL}$, indicating no abnormalities of anemia, thrombocytopenia, and coagulopathy. We did not investigate alternative causes, such as acquired hemophilia or acquired vitamin $\mathrm{K}$ deficiency. The patient had a medical history of hypertension and cerebral infarction without any prescription. He wanted to receive favipiravir and tocilizumab to manage COVID-19 after receiving informed consent despite the unproven effect of these medications on COVID-19. During his ICU stay, newonset paroxysmal atrial fibrillation (Af) occurred. The CHADS2 VASC score was 5 points (age, stroke, hypertension) and HAS-BLED was 2 points (age, stroke). After the fall, there was no obvious hematoma on the body surface, and the $\mathrm{Hb}$ level was normal at the time of the visit; therefore, we considered that there was no active bleeding. We decided to start heparin aiming for aPTT between 42 and 56 seconds (1.5 to 2.0 times compared to aPTT standard $[28.0 \mathrm{~s}])$. As a result, the heparin dose increased up to 24.000 units. In addition, we administered ART-123, a recombinant human soluble thrombomodulin (rTM), considering the hypercoagulation status (Figure 1). On day 5 after admission, his respiratory condition improved, and he was extubated. However, an anterior chest hematoma was observed the following day. The $\mathrm{Hb}$ level dropped from $11 \mathrm{mg} / \mathrm{dL}$ to $7 \mathrm{mg} / \mathrm{dL}$, suggesting continuous bleeding. The platelet count was within the normal range. A clinical decision for CT was made to detect the source of the hematoma. Computed tomography

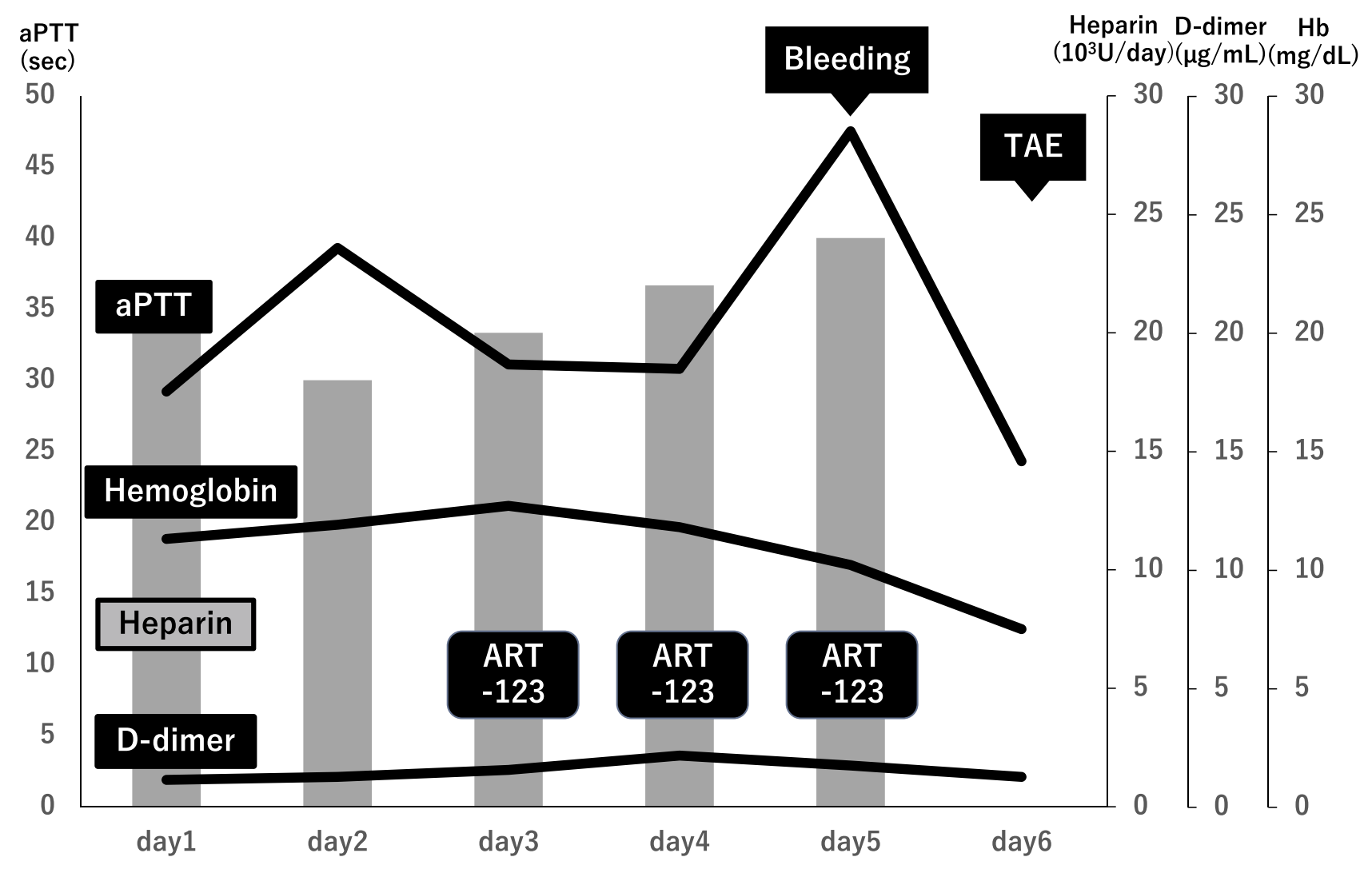

Figure I Clinical course.

Abbreviations: Hb, hemoglobin (Bar graph); aPTT, activated partial thromboplastin time; ART-I23, recombinant human soluble thrombomodulin; TAE, transcatheter embolization. 
(CT) revealed multiple hematomas, including a massive chest hematoma (Figure 2A and B) with active extravasation and an obturatorius internus muscle hematoma (Figure 2C). An emergency TAE was indicated, and the angiography suite was prepared prior to patient transfer. The walls and equipment were covered with vinyl to prevent contamination, and the suite was set to a negative pressure of $-5 \mathrm{~Pa}$. Maximum barrier precautions (caps, masks, eye guards, sterile gowns, sterile gloves, double glove use, and N95 respirator masks) were employed by all the hospital staff involved. TAE was approached from the right common femoral artery. Digital subtraction angiography (DSA) of the left axillary artery was performed. Extravasation was observed at the acromial branch of the left thoracoacromial artery (Figure $3 \mathrm{~A})$. The second source of extravasation was the left lateral thoracic artery, which is also a branch of the axillary artery (Figure 3B). Extravasations from these arteries were embolized using gelatin sponge and coil. Hemostasis after TAE was confirmed using DSA of the subclavian artery. After TAE, the bruising of the chest wall subsided. Seven days after TAE, the patient was discharged from the ICU. A 22-day follow-up CT showed that the chest hematoma had decreased in size and there were no signs of extravasation or pseudoaneurysm. The patient was transferred to another hospital the following day.

\section{Discussion}

Coagulopathy is a common feature in patients with COVID-19. The pathogenesis of COVID-19-induced coagulopathy has not yet been fully elucidated, but the mechanisms may overlap with those of sepsis-associated
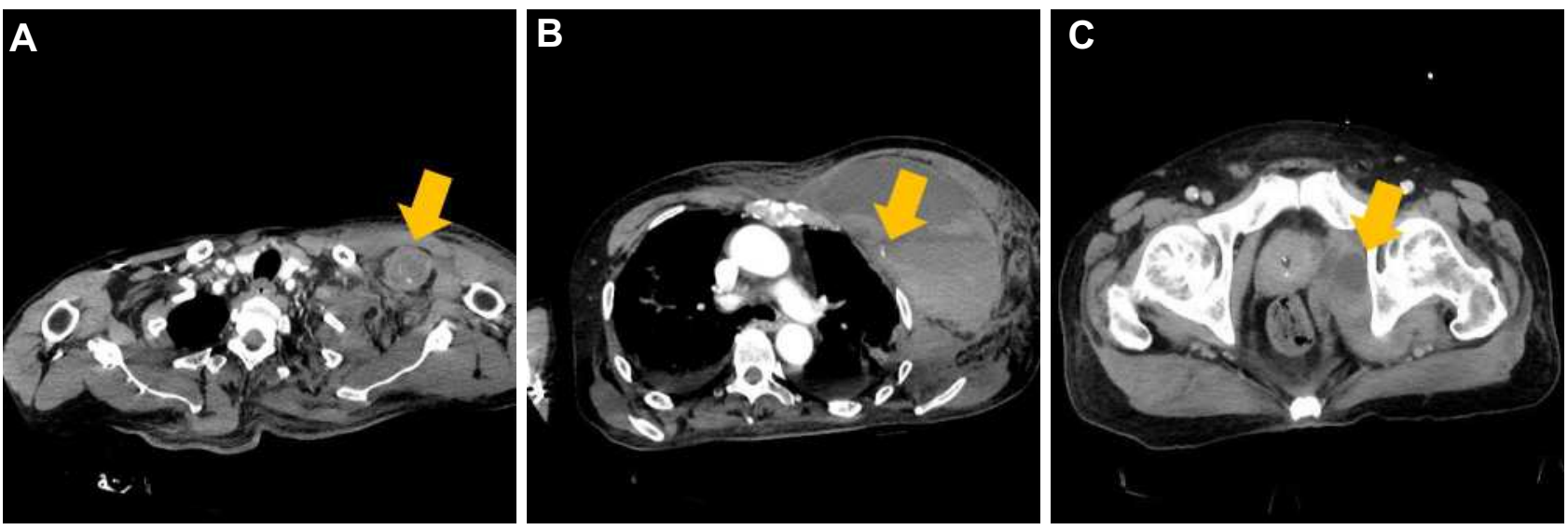

Figure 2 Chest computed tomography (CT). CT showing multiple hematomas, including a chest wall hematoma with active extravasation (A, B) and an obturatorius internus muscle hematoma (C). The arrow indicates extravasation.
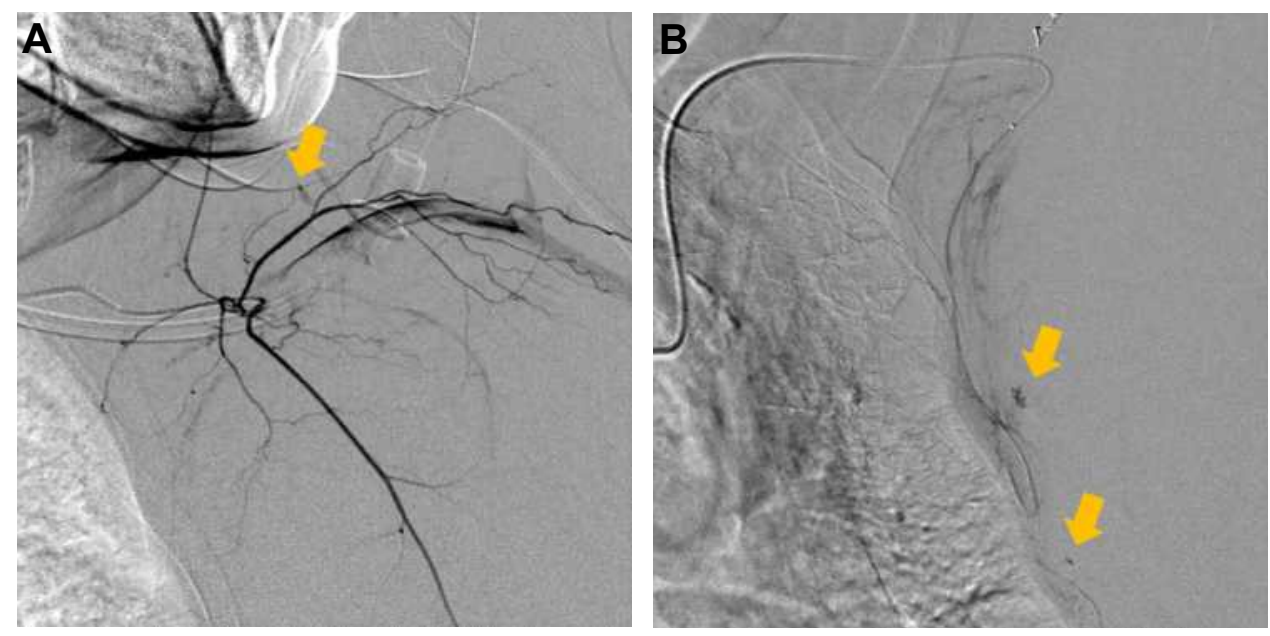

Figure 3 Transcatheter embolization. (A) Extravasation from the left thoracoacromial artery. (B) Extravasation from the branch of the left lateral thoracic artery. The arrow indicates extravasation. 
coagulopathy (SAC). The cytokines storm, increased levels of damage-associated molecular patterns (DAMPs), the stimulation of cell-death mechanisms and vascular endothelial damage are the major causes of coagulation disorder in any severe infection, including bacterial and viral infections. ${ }^{2}$ It is characterized by a hypercoagulable state with a high D-dimer (DD) level in COVID-19 patients. ${ }^{2-4}$ Considering that COVID-19 can induce a hypercoagulable state, heparin has been considered as one of the therapeutic options for patients diagnosed with COVID-19. ${ }^{5}$ In addition, a recent study demonstrated that heparin is considered to have antiinflammatory properties, which may provide an added benefit to patients with COVID-19. ${ }^{6}$ In the current case, we administered the therapeutic dose of heparin, aiming both effects on anti-thrombotic prophylaxis for Af and COVID-19 associated coagulopathy. However, the American Society of Hematology (ASH) recommends an anti-thrombotic prophylaxis dose rather than a therapeutic dose, and there is a report that using a therapeutic dose of heparin increases bleeding complications. ${ }^{7}$ Therefore, we have to pay careful attention to the dose of heparin for patients with Af.

In the current case, we also administered ART-123 to investigate its effect on COVID-19 associated hypercoagulation status, which may be a mechanism similar to that of SAC. The effect of ART-123 was recently reported by Vincent et al. Among patients with SAC, administration of ART-123, compared with placebo, did not significantly reduce 28-day all-cause mortality in a randomized, double-blind, placebo-controlled, multinational, multicenter, parallel-group Phase 3 study named the Sepsis Coagulopathy Asahi Recombinant LE Thrombomodulin: SCARLET) trial. ${ }^{8}$ However, approximately $20 \%$ of patients did not meet the criteria for coagulopathy as defined by the international normalized ratio (INR) and platelet count at baseline in this study. Therefore, Levi et al analyzed the effect of ART-123 on 28-day all-cause mortality in subgroups categorized by baseline coagulation biomarker levels (prothrombin fragments 1.2, thrombin-antithrombin complex, D-dimer) in patients with SAC in the SCARLET trial. They concluded that compared with patients receiving placebo, patients treated with recombinant human soluble thrombomodulin having higher baseline thrombin generation biomarker levels had lower mortality. ${ }^{9}$ However, a previous report demonstrated that ART-123 causes bleeding complications when used in combination with heparin. ${ }^{10}$ Furthermore, the T1/2 (half-life) of ART-123 is about 20 hours, which is longer than that of heparin; therefore, caution is required when administering ART-123. ${ }^{11}$ In addition, elderly people may easily develop bleeding complications due to the fragility of their tissues and blood vessels. Heparin and ART-123 for the treatment of coagulopathy in elderly COVID-19 patients with a history of traumatic injury should be administered with caution considering the potential bleeding complications.

In the current case, favipiravir and tocilizumab were administered before publishing evidence of the benefit of these medications at that time. Recently, these drugs have not been routinely recommended for patients who require mechanical ventilation due to the lack of data showing benefits at this advanced stage of the disease, considering the NIH COVID-19 Treatment Guidelines. ${ }^{12}$

In this case, the cause of the bleeding complications and indication of treatment might not be fully proven, as described above. It was necessary to carefully consider the indication and administration of ART-123 and heparin to avoid harm to the patient.

\section{Conclusion}

We encountered a case of multiple hematomas, including a massive chest wall hematoma due to bleeding from the branches of the axial arteries in a COVID-19 patient for several reasons (fall, heparin, and ART-123). The patient successfully underwent emergency TAE. When anticoagulants are considered in the management of Af and COVID19 , it is necessary to closely monitor potential bleeding complications and prevent patient harm.

\section{Informed Consent}

Informed consent for publication of clinical details and images was obtained from the patient.

\section{Acknowledgments}

The authors thank Nao Urushibata, MD, Jun Aoki, MD, and Marie Takahashi, M.D., for their helpful comments.

\section{Approval of the Research Protocol}

Institutional approval was not required to publish the case details.

\section{Disclosure}

The authors declare no conflicts of interest for this article. 


\section{References}

1. World Health Organization. Coronavirus disease (COVID-19) pandemic. Available from: https://www.who.int/emergencies/diseases/ novel-coronavirus-2019. Accessed March 20, 2021.

2. Iba T, Levy JH, Levi M, Connors JM, Thachil J. Coagulopathy of coronavirus disease 2019. Crit Care Med. 2020;10:1097.

3. Chen T, Wu D, Chen H, et al. Clinical characteristics of 113 deceased patients with coronavirus disease 2019: retrospective study. BMJ. 2020;368:m1091. doi:10.1136/bmj.m1091

4. Helms J, Tacquard C, Severac F, et al. High risk of thrombosis in patients with severe SARS-CoV-2 infection: a multicenter prospective cohort study. Intern Care Med. 2020;46:1089-1098. doi:10.1007/ s00134-020-06062-x

5. Thachil J, Tang N, Gando S, et al. ISTH interim guidance on recognition and management of coagulopathy in COVID-19. J Thromb Haemost. 2020;18:1023-1026. doi:10.1111/jth.14810

6. Poterucha TJ, Libby P, Goldhaber SZ, et al. More than an anticoagulant: do heparins have direct anti-inflammatory effects? Thromb Haemost. 2017;117:437-444. doi:10.1160/TH16-08-0620

7. Musokea N, Loa KB, Albano J, et al. Anticoagulation and bleeding risk in patients with COVID-19. Thromb Res. 2020;196:227-230. doi:10.1016/j.thromres.2020.08.035
8. Vincent JL, Francois B, Zabolotskikh I, et al. Effect of a recombinant human soluble thrombomodulin on mortality in patients with Sepsis-Associated Coagulopathy. JAMA. 2019;321:1993-2002. doi:10.1001/jama.2019.5358

9. Levi M, Vincent JL, Tanaka K, et al. Effect of a recombinant human soluble thrombomodulin on baseline coagulation biomarker levels and mortality outcome in patients with Sepsis-Associated Coagulopathy. Crit Care Med. 2020;48(8):1140-1147. doi:10.1097/ CCM.0000000000004426

10. Takahashi H, Shoko T, Kuriyama K, et al. Characteristic of the patients with hemorrhagic events after recombinant thrombomodulin administration for sepsis-induced DIC. KANTO J Jpn Assoc Acute Med. 2016;37(2):412-415.

11. Nakashima M, Kanamaru M, Umemura K, Tsuruta $K$. Pharmacokinetics and safety of a novel recombinant soluble human thrombomodulin, ART-123, in healthy male volunteers. J Clin Pharmacol. 1998;38:40-44. doi:10.1002/j.1552-4604.1998. tb04375.x

12. Therapeutic Management of Adults With COVID-19. NIH COVID-19 treatment guidelines. Available from: https://www.cov id19treatmentguidelines.nih.gov/therapeutic-management/. Accessed March 20, 2021.

\section{Publish your work in this journal}

The Open Access Emergency Medicine is an international, peerreviewed, open access journal publishing original research, reports, editorials, reviews and commentaries on all aspects of emergency medicine. The manuscript management system is completely online and includes a very quick and fair peer-review system, which is all easy to use. Visit http://www.dovepress.com/testimonials.php to read real quotes from published authors. 